Brazilian Journal
of Chemical
Engineering

ISSN 0104-6632

Printed in Brazil

www.abeq.org.br/bjche

Vol. 29, No. 01, pp. 159 - 166, January - March, 2012

\title{
COMPUTATIONAL MODELLING OF MHD UNSTEADY FLOW AND HEAT TRANSFER TOWARD A FLAT PLATE WITH NAVIER SLIP AND NEWTONIAN HEATING
}

\author{
O. D. Makinde \\ Institute for Advance Research in Mathematical Modelling and Computations, \\ Cape Peninsula University of Technology, P. O. Box 1906, Bellville 7535, South Africa. \\ E-mail: makinded@cput.ac.za \\ (Submitted: June 4, 2011 ; Revised: September 20, 2011 ; Accepted: October 1, 2011)
}

\begin{abstract}
The combined effects of Navier slip and Newtonian heating on an unsteady hydromagnetic boundary layer stagnation point flow towards a flat plate in the presence of a magnetic field are studied. The self-similar equations are obtained using similarity transformations and solved numerically by a shooting algorithm with a Runge-Kutta Fehlberg integration scheme. The velocity profiles, temperature profiles, the local skin friction coefficient, and the local Nusselt number are computed and discussed in details for various values of the different parameters. Numerical results are presented both in tabular and graphical forms, illustrating the effects of these parameters on the thermal and concentration boundary layers. It is revealed that the thermal boundary layer thickens with a rise in the flow unsteadiness and as Newtonian heating intensifies, while the local skin friction and the rate of heat transfer at the plate surface change significantly due to the slip parameter.

Keywords: Unsteady flow; Flat plate; Navier slip; Newtonian heating; Magnetic field; Similarity solution.
\end{abstract}

\section{INTRODUCTION}

Analysis of unsteady hydromagnetic boundary layer flow and heat transfer of electrically conducting fluids is of great interest in many branches of engineering. Practical applications are found in the design of cooling systems for electronic devices, in the field of solar energy collection, geothermal reservoirs, heat exchangers, cooling of an infinite metallic plate in a cooling bath, magnetohydrodynamic (MHD) stirring of molten metal, magnetic-levitation casting, MHD marine propulsion, the boundary layer along a liquid film in condensation processes, and a polymer sheet or filament extruded continuously from a dye. Considerable reviews of this area have been made by many researchers such as Chamkha and Khaled
(2000), Makinde and Ogulu (2008), Beg et al. (2009), Makinde (2010), etc. Vajravelu and Nayfeh (1992) discussed the hydromagnetic flow of a dusty fluid over a stretching sheet. The MHD heat and mass transfer in a flow of viscous incompressible fluid past an infinite vertical plate has been studied by Singh and Singh (2003). Ali and Magyari (2007) reported a numerical solution for unsteady boundary layer flow and heat transfer induced by a submerged stretching surface while its steady motion is slowed down gradually. An unsteady hydromagnetic free convection flow of elastico-viscous fluid past an infinite vertical plate taking into account the Hall effect has been investigated by Chaudhary and Jha (2008). A computational analysis of MHD boundarylayer flow and mass transfer past a vertical plate in a porous medium with constant heat flux was

*To whom correspondence should be addressed 
presented by Makinde (2009). Dulal and Hiremath (2009) investigated the heat transfer over an unsteady stretching surface embedded in a porous medium. In spite of the importance of these MHDrelated studies on boundary layer flow problems, the possibility of fluid exhibiting apparent slip phenomenon on the solid surface has received little attention. The no-slip condition at the fluid-solid interface is a hypothesis rather than a condition deduced from any principle, and thus its validity has been continuously debated in the scientific literature (Choi et al., 2002). Meanwhile, many experimental results have provided evidence to support the slip condition (Pit et al., 2000; Huang and Breuer, 2007). In a pioneering work, Navier (1823) introduced a more general boundary condition, namely the fluid velocity component tangential to the solid surface, relative to the solid surface, is proportional to the shear stress on the fluid-solid interface. The proportionality is called the slip length, which describes the slipperiness of the surface. Matthews and Hills (2008) investigated numerically the effects of slip on momentum boundary layer thickness on a flat plate. Recently, Bhattacharyya et al. (2011) presented numerical results on the effects of velocity slip on hydromagnetic boundary layer flow and heat transfer over a flat plate. The combined effects of Hall current and wall slip on unsteady MHD flow of a viscoelastic fluid past an infinite vertical porous plate through a porous medium was investigated by Kumar and Chand (2011).

The objective of this study is to determine the combined effects of Navier slip and Newtonian heating on an unsteady hydromagnetic boundary layer flow over a flat surface. In the subsequent sections the classical similarity reductions of the boundary layer equations are derived and the resulting ordinary differential equations are solved numerically using shooting algorithm together with a Runge-Kutta Fehlberg integration scheme. Finally, we present a discussion of the results and we make some concluding remarks.

\section{MATHEMATICAL MODEL}

Consider an unsteady two-dimensional magnetohydrodynamic boundary layer stagnation point flow with heat transfer and Navier slip towards a flat plate. The lower surface of the plate is assumed to be heated by convection from a hot fluid at temperature $\mathrm{T}_{\mathrm{f}}$, which provides a heat transfer coefficient $h_{f}$, while the upper surface is subjected to a stream of an electrically conducting cold fluid at temperature $T_{\infty}$ in the presence of magnetic field of strength $\mathrm{B}_{0}$ imposed along the $\mathrm{y}$-axis, as shown in Fig. 1. The induced magnetic field due to the motion of the electrically conducting fluid is negligible. It is also assumed that the external electrical field is zero and that the electric field due to the polarization of charges is negligible.

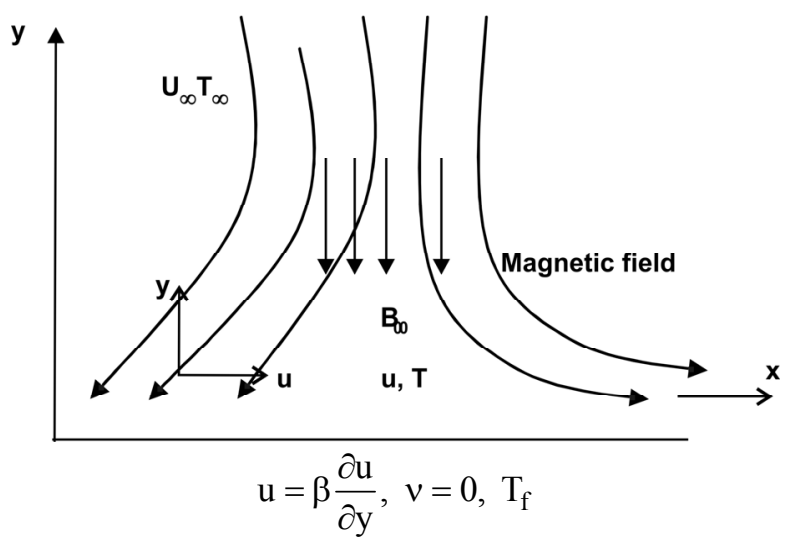

Figure 1: Flow configuration and coordinate system

Under the usual boundary layer approximation, the continuity, momentum, and energy equations describing the flow can be written as (Vajravelu and Nayfeh, 1992; Dulal and Hiremath, 2009):

$\frac{\partial \mathrm{u}}{\partial \mathrm{x}}+\frac{\partial \mathrm{v}}{\partial \mathrm{y}}=0$

$\frac{\partial u}{\partial t}+u \frac{\partial u}{\partial x}+v \frac{\partial u}{\partial y}=v \frac{\partial^{2} u}{\partial y^{2}}-$

$\frac{\sigma \mathrm{B}_{\mathrm{O}}^{2}\left(\mathrm{u}-\mathrm{U}_{\infty}\right)}{\rho}+\frac{(\lambda+\mathrm{c}) \mathrm{U}_{\infty}}{1-\lambda \mathrm{t}}$

$\frac{\partial \mathrm{T}}{\partial \mathrm{t}}+\mathrm{u} \frac{\partial \mathrm{T}}{\partial \mathrm{x}}+\mathrm{v} \frac{\partial \mathrm{T}}{\partial \mathrm{y}}=\alpha \frac{\partial^{2} \mathrm{~T}}{\partial \mathrm{y}^{2}}$,

where the free stream velocity is given as (Bhattacharyya et al., 2011):

$\mathrm{U}_{\infty}=\frac{\mathrm{xc}}{1-\lambda \mathrm{t}}$,

where $\mathrm{c}$ represents the straining parameter due to stagnation point flow of the fluid towards the plate surface, as shown in Fig.1 . The boundary conditions at the plate surface and far into the cold fluid may be written as: 
$u(t, x, 0)=\beta \frac{\partial u}{\partial y}(t, x, 0), v(t, x, 0)=0$

$-\mathrm{k} \frac{\partial \mathrm{T}}{\partial \mathrm{y}}(\mathrm{t}, \mathrm{x}, 0)=,\mathrm{h}_{\mathrm{f}}\left[\mathrm{T}_{\mathrm{f}}-\mathrm{T}(\mathrm{t}, \mathrm{x}, 0)\right]$

$\mathrm{u}(\mathrm{t}, \mathrm{x}, \infty)=\mathrm{U}_{\infty}, \mathrm{T}(\mathrm{t}, \mathrm{x}, \infty)=\mathrm{T}_{\infty}$.

The situation $\beta=0$ corresponds to no-slip, while full lubrication is described in the limit $\beta \rightarrow \infty$. The stream function $\psi$, satisfies the continuity Eq. (1) automatically, with:

$\mathrm{u}=\frac{\partial \psi}{\partial \mathrm{y}}$ and $\mathrm{v}=-\frac{\partial \psi}{\partial \mathrm{x}}$.

In order to simplify the mathematical analysis of the problem, we introduce the following dimensionless variables:

$\eta=y \sqrt{\frac{c}{v(1-\lambda t)}}, \quad \psi=x \sqrt{\frac{c v}{(1-\lambda t)}} f(\eta)$,

$\theta(\eta)=\frac{T-T_{\infty}}{T_{f}-T_{\infty}}$

Substituting Eq. (7) into Eqs. (1)-(6), we obtain:

$$
\begin{aligned}
& f^{\prime \prime \prime}+f f "-f^{\prime 2}-A\left(f^{\prime}+\frac{\eta}{2} f^{\prime \prime}-1\right)- \\
& \operatorname{Ha}\left(f^{\prime}-1\right)+1=0 \\
& \theta^{\prime \prime}+\operatorname{Pr}\left(f \theta^{\prime}-\frac{A}{2} \eta \theta^{\prime}\right)=0, \\
& f(0)=0, f^{\prime}(0)=\delta f^{\prime \prime}(0), \quad \theta^{\prime}(0)=\operatorname{Bi}[\theta(0)-1], \\
& f^{\prime}(\infty)=1, \quad \theta(\infty)=0,
\end{aligned}
$$

where the prime symbol represents the derivative with respect to $\eta$ and:

$$
\mathrm{Ha}=\frac{\sigma \mathrm{B}_{0}^{2}}{\rho \mathrm{c}}(1-\lambda \mathrm{t})
$$

(the local magnetic field parameter),

$$
\mathrm{Bi}=\frac{\mathrm{h}_{\mathrm{f}}}{\mathrm{k}} \sqrt{\frac{\mathrm{U}(1-\lambda \mathrm{t})}{\mathrm{c}}}
$$

(the local Biot number),
$\mathrm{A}=\frac{\lambda}{\mathrm{c}}$

(Unsteadiness parameter),

$\operatorname{Pr}=\frac{v}{\alpha}$

(the Prandtl number),

$\delta=\beta \sqrt{\frac{c}{v(1-\lambda t)}}$

(the local slip parameter).

The set of Equations (8)-(9) under the boundary conditions (10)-(11) have been solved numerically using a shooting algorithm with a Runge-Kutta Fehlberg integration scheme (Nachtsheim and Swigert, 1965). From the process of numerical computation, the plate surface temperature, the skinfriction coefficient and the Nusselt number, which are respectively proportional to $\theta(0), f^{\prime \prime}(0)$ and $-\theta^{\prime}(0)$, are also worked out and their numerical values are presented in a tabular form. The accuracy of this numerical method was validated by direct comparison with the numerical results reported by Dulal and Hiremath (2009) for the unsteady boundary layer flow over a moving plate in the absence of a magnetic field $(\mathrm{Ha}=0)$, Navier slip $(\delta=0)$ and Newtonian heating $(\mathrm{Bi}=0)$ modelled as:

$$
\begin{aligned}
& f^{\prime \prime \prime}+f f "-f^{\prime 2}-A\left(f^{\prime}+\frac{\eta}{2} f^{\prime \prime}\right)=0 \text { with } \\
& f(0)=0, f^{\prime}(0)=1, f^{\prime}(\infty)=0
\end{aligned}
$$

and a perfect agreement is observed, as demonstrated in Table 1 below;

Table 1: Computations showing comparison with Dulal and Hiremath (2009) results for $\delta=0$, $\mathbf{H a}=\mathbf{0}$.

\begin{tabular}{|c|c|c|}
\hline $\mathbf{A}$ & $\begin{array}{c}\mathbf{f}^{\prime \prime}(\mathbf{0}) \\
\mathbf{D}-\mathbf{H ~ ( 2 0 0 9 )}\end{array}$ & $\begin{array}{c}\mathbf{f}^{\prime \prime}(\mathbf{0}) \\
\text { Present }\end{array}$ \\
\hline 0.5 & -1.167221 & -1.167221 \\
1.0 & -1.320540 & -1.320540 \\
1.5 & -1.459687 & -1.459687 \\
2.0 & -1.587403 & -1.587403 \\
\hline
\end{tabular}

Also, we conducted a direct comparison with the numerical results reported by Bhattacharyya et al. (2011) for the unsteady boundary layer stagnationpoint flow towards a stretching sheet with slip in the absence of magnetic field $(\mathrm{Ha}=0)$ modelled as: 
$f^{\prime \prime}+f f "-f^{\prime 2}-A\left(f^{\prime}+\frac{\eta}{2} f^{\prime \prime}-R\right)+R^{2}=0$

with $f(0)=0, f^{\prime}(0)=1+\delta f^{\prime \prime}, f^{\prime}(\infty)=R$,

where $\mathrm{R}$ is the parameter representing the velocity ratio between the stretching sheet and that of the free stream. The results in Table 2 below agree almost perfectly with those of Bhattacharyya et al. (2011).

Table 2: Computations showing comparison with Bhattacharyya et al. (2011) results for $\mathrm{Ha}=\mathrm{A}=\delta=0$.

\begin{tabular}{|c|c|c|}
\hline $\mathbf{R}$ & $\begin{array}{c}\mathbf{f}^{\prime \prime}(\mathbf{0}) \\
\text { Bhattacharyya } \text { et al. } \\
\mathbf{( 2 0 1 1 )}\end{array}$ & $\begin{array}{c}\mathbf{f}^{\prime \prime}(\mathbf{0}) \\
\text { Present }\end{array}$ \\
\hline 0.1 & -0.969386 & -0.9693871 \\
0.2 & -0.918107 & -0.918110 \\
0.5 & -0.667263 & -0.667263 \\
2.0 & 2.017503 & 2.017510 \\
3.0 & 4.729284 & 4.729283 \\
\hline
\end{tabular}

\section{RESULTS AND DISCUSSION}

The numerical computation results are demonstrated in Table 3 and Figures 2-8. In order to have greater insight into the qualitative analysis of the results, we have taken the values of various parameters controlling the flow systems as 0.72 (Air) $\leq \operatorname{Pr} \leq 7.1$ (Water), $0 \leq \mathrm{A} \leq 1,0 \leq \delta \leq 5,0.1 \leq \mathrm{Bi} \leq 1$, $0 \leq \mathrm{Ha} \leq 5$. Table 3 illustrates the effects of thermophysical parameters on the local skin friction coefficient and local Nusselt number. It is noteworthy that the temperature gradient $\theta^{\prime}(0)$ is negative for all parameter values considered in this study. This simply implies that the heat flow is from the hot fluid at the lower surface of the plate to the cold fluid on the upper surface of the plate. Moreover, it is interesting to note that the local heat transfer rate at the plate surface increases with increasing values of $\mathrm{Bi}, \mathrm{Ha}, \operatorname{Pr}, \delta$ and decreases with increasing values of $A$. This can be attributed to the fact that the magnitude of the temperature gradient at the plate surface increases with an increase in local Biot number, magnetic field intensity, Prandtl number and slip length. Meanwhile, the effect of Navier slip parameter $(\delta)$ is to decrease the local skin friction. We also note that the local skin friction increases with an increase in the magnetic field intensity and flow unsteadiness.

\section{Effects of Parameter Variation on Velocity Profiles}

The illustrations of the velocity profiles with respect to the transverse distance are displayed in Figures 2-4. Generally, the fluid velocity is lowest at the plate surface and increases gradually to its free stream values satisfying the far field boundary condition. For the set of parameter values utilised, it is interesting to note that the far field conditions for velocity profiles are satisfied at a transverse distance $\eta=3$. However, this is not the case for temperature profiles with the same set of parameter values. From Figure 2, we observe that the fluid velocity at the plate surface increases with an increase in the slip parameter $(\delta)$. This is consistent with the fact that higher $\delta$ means an increase in the lubrication and slipperiness of the surface. In Figure 3 , a slight increase in the fluid velocity towards the plate surface is observed with an increase in the flow unsteadiness. An increase in the magnetic field intensity Ha causes an overshoot of the fluid velocity towards the plate surface.

Table 3: Computation showing $f^{\prime \prime}(0), \theta(0)$ and $\theta^{\prime}(0)$ for various values of key parameters

\begin{tabular}{|c|c|c|c|c|c|c|c|}
\hline $\mathbf{B i}$ & $\mathbf{H a}$ & $\mathbf{A}$ & $\boldsymbol{\delta}$ & $\mathbf{P r}$ & $\mathbf{f}^{\prime \prime}(\mathbf{0})$ & $\mathbf{\theta}^{\prime}(\mathbf{0})$ & $\mathbf{\theta}(\mathbf{0})$ \\
\hline 0.1 & 0.1 & 0.1 & 0.1 & 0.72 & 1.186380878 & 0.08358870 & 0.16411293 \\
0.5 & 0.1 & 0.1 & 0.1 & 0.72 & 1.186380878 & 0.25231253 & 0.49537492 \\
1.0 & 0.1 & 0.1 & 0.1 & 0.72 & 1.186380878 & 0.33745722 & 0.66254277 \\
0.1 & 0.5 & 0.1 & 0.1 & 0.72 & 1.296927194 & 0.08383121 & 0.16168785 \\
0.1 & 1.0 & 0.1 & 0.1 & 0.72 & 1.418129883 & 0.08406929 & 0.15930705 \\
0.1 & 0.1 & 0.5 & 0.1 & 0.72 & 1.267686514 & 0.08128091 & 0.18719082 \\
0.1 & 0.1 & 1.0 & 0.1 & 0.72 & 1.361534490 & 0.07638725 & 0.23612748 \\
0.1 & 0.1 & 0.1 & 1.0 & 0.72 & 0.603133607 & 0.08563567 & 0.14364329 \\
0.1 & 0.1 & 0.1 & 3.0 & 0.72 & 0.275659634 & 0.08635366 & 0.13646331 \\
0.1 & 0.1 & 0.1 & 0.1 & 1.00 & 1.186380878 & 0.08534327 & 0.14656723 \\
0.1 & 0.1 & 0.1 & 0.1 & 3.00 & 1.186380878 & 0.09000268 & 0.09997318 \\
0.1 & 0.1 & 0.1 & 0.1 & 7.10 & 1.186380878 & 0.09262444 & 0.07375555 \\
\hline
\end{tabular}




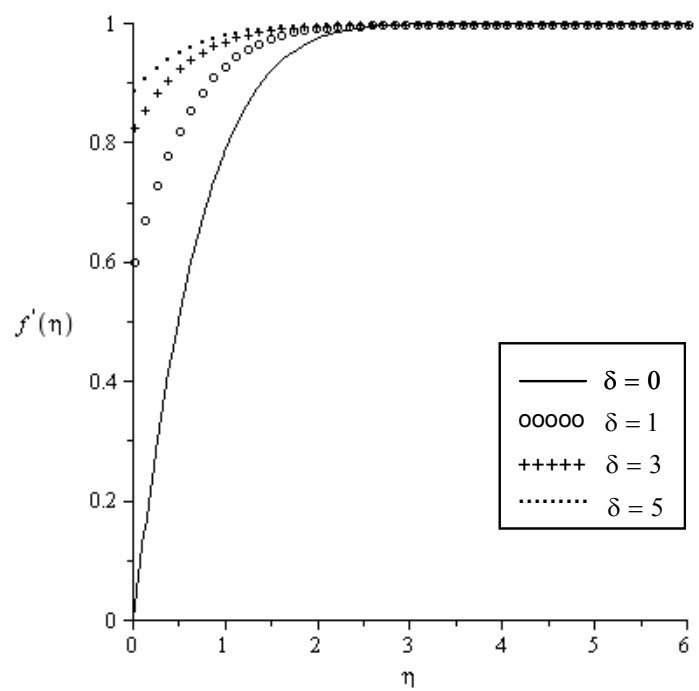

Figure 2: Velocity profiles for $\operatorname{Pr}=0.72$, $\mathrm{A}=0.1, \mathrm{Bi}=0.1, \mathrm{Ha}=0.1$.

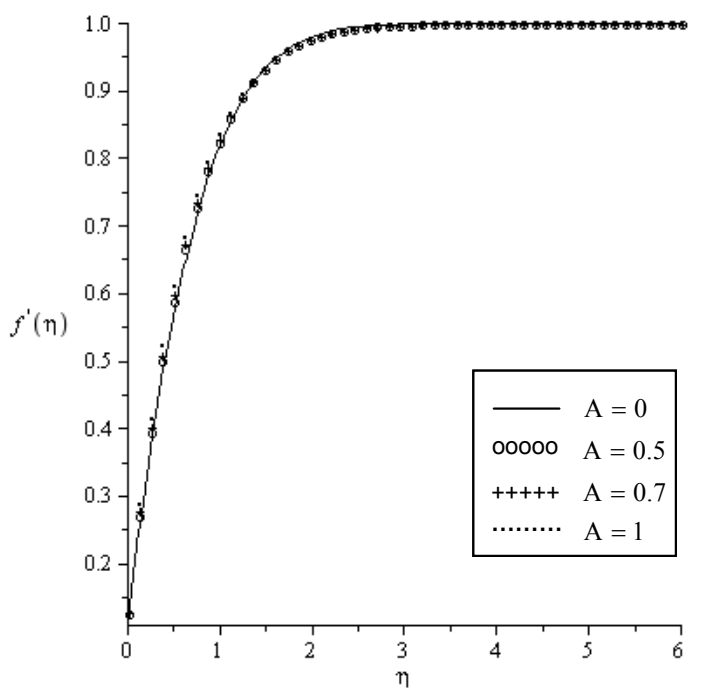

Figure 3: Velocity profiles for $\operatorname{Pr}=0.72$, $\delta=0.1, \mathrm{Ha}=0.1, \mathrm{Bi}=0.1$

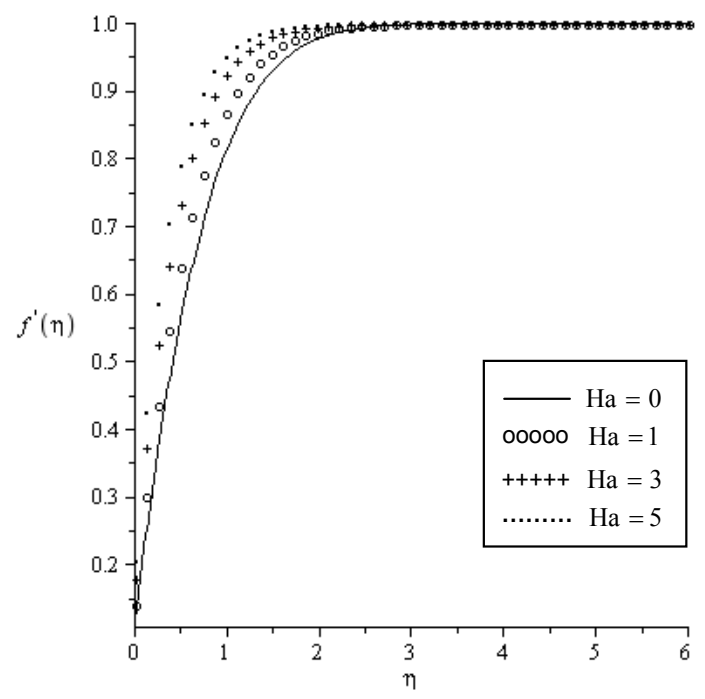

Figure 4: Velocity profiles for $\operatorname{Pr}=0.72, \delta=0.1, \mathrm{~A}=0.1, \mathrm{Bi}=0.1$

\section{Effects of Parameter Variation on Temperature Profiles}

Figures 5 and 6 show the effect of the Newtonian heating and the flow unsteadiness on the temperature profiles. The maximum value of fluid temperature is attained at the plate surface and decreases exponentially to the free stream zero value away from the plate, satisfying the boundary condition. It is seen that, in both cases, the thermal boundary layer thickness increases with an increase in the local Biot number $(\mathrm{Bi})$ and the unsteadiness parameter (A). This can be attributed to the fact that, as $\mathrm{Bi}$ increases, the heat transfer rate from the hot fluid at the lower side of the plate to the cold fluid at the upper side increases. This results in an elevation of the fluid temperature at the upper side. The effects of the slip parameter and Prandtl number on the temperature profiles are plotted in Figures 7 and 8. It is evident from these figures that the presence of the surface slipperiness affects the temperature of the fluid inversely. This can be seen clearly from the temperature curves, which decrease as the slip parameter $\delta$ increases. A similar trend is observed with an increase in the Prandtl number Pr. The thermal boundary layer thickness decreases as the Prandtl number increases from 0.72 (Air) to 7.1 (Water) due to a decrease in the fluid thermal diffusivity. This is in agreement with the physical fact that, at higher Prandtl number, the fluid has a thinner thermal boundary layer and this increases the gradient of temperature. 


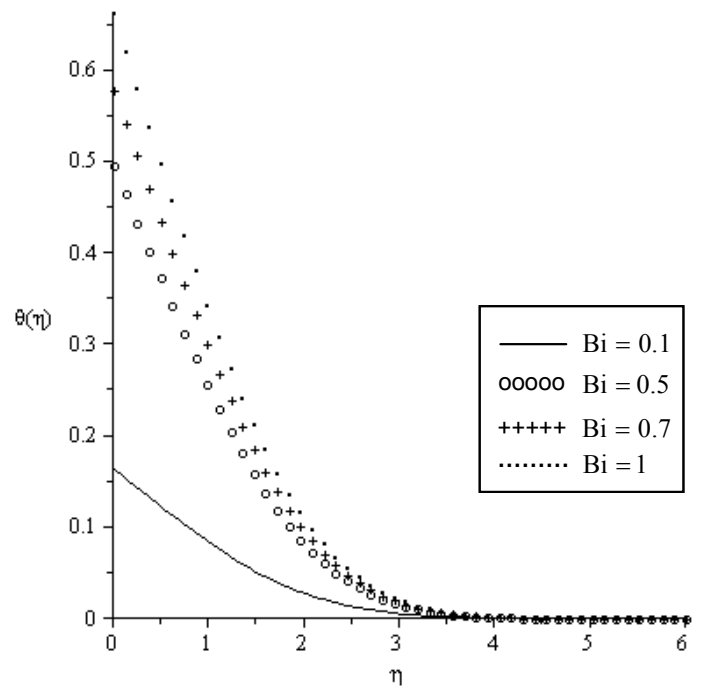

Figure 5: Temperature profiles for $\operatorname{Pr}=0.72$, $\mathrm{A}=0.1, \delta=0.1, \mathrm{Ha}=0.1$

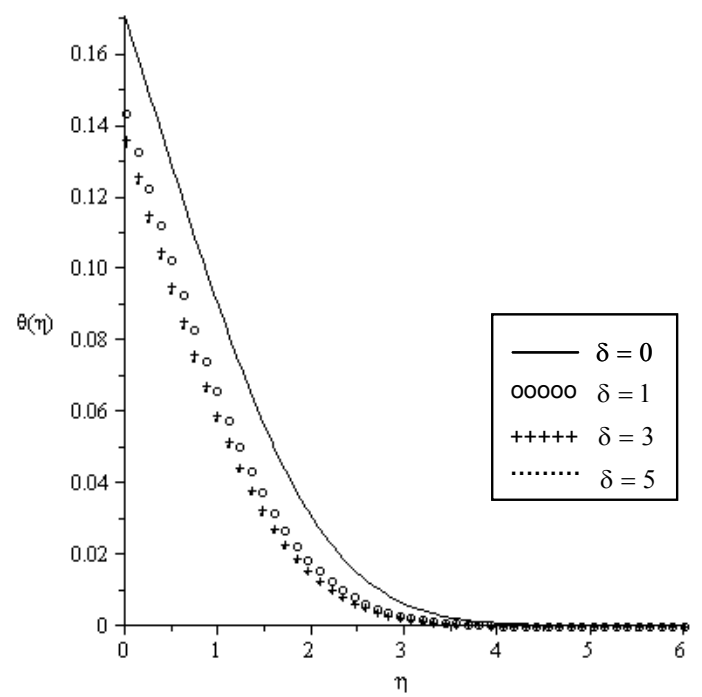

Figure 7: Temperature profiles for $\operatorname{Pr}=0.72$, $\mathrm{A}=0.1, \mathrm{Bi}=0.1, \mathrm{Ha}=0.1$.

\section{CONCLUSIONS}

The problem of unsteady hydromagnetic boundary layer stagnation point flow towards a flat plate with Navier slip and Newtonian heating was studied. The governing equations were developed and transformed into a self-similar form and solved numerically by a shooting algorithm with a RungeKutta Fehlberg integration scheme. Our results revealed that the fluid velocity increases, while the local skin friction decreases, with an increase in the slip parameter $(\delta)$. The thermal boundary layer

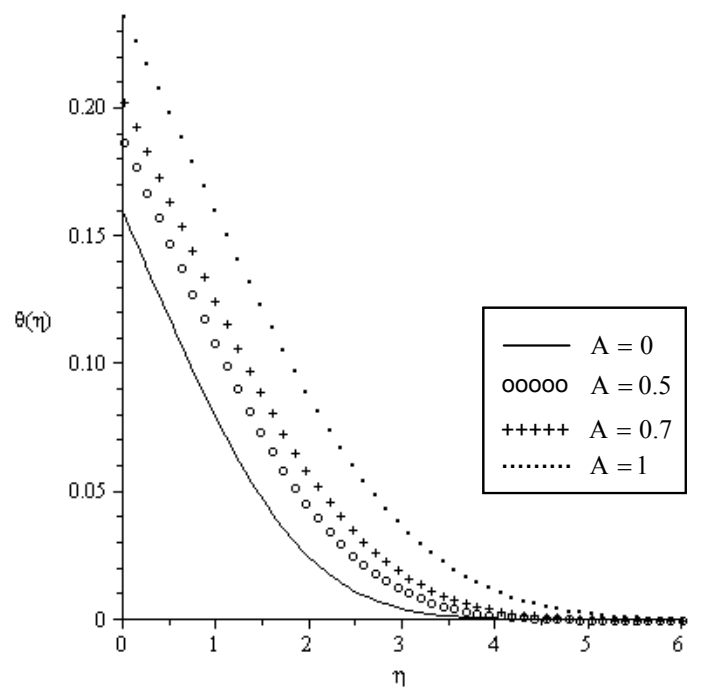

Figure 6: Temperature profiles for $\operatorname{Pr}=0.72$, $\delta=0.1, \mathrm{Ha}=0.1, \mathrm{Bi}=0.1$

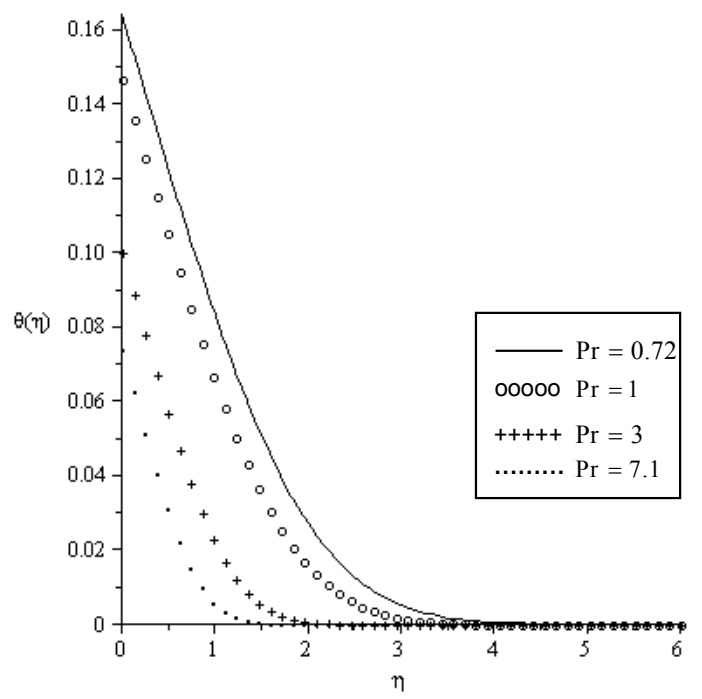

Figure 8: Temperature profiles for $\mathrm{Ha}=0.1$, $\delta=0.1, \mathrm{~A}=0.1, \mathrm{Bi}=0.1$

thickness is enhanced by increasing the intensity of Newtonian heating (Bi) and flow unsteadiness (A), while a decreases in the thermal boundary layer thickness is observed with an increase in the velocity slip $(\delta)$ and Prandtl number (Pr).

\section{ACKNOWLEDGEMENTS}

The author would like to thank the National Research Foundation of South Africa Thuthuka programme for financial support. 


\section{NOMENCLATURE}

$(\mathrm{u}, \mathrm{v})$

$(\mathrm{x}, \mathrm{y})$

$\mathrm{B}_{0}$

$\operatorname{Pr}$

Bi

$\mathrm{T}_{\infty}$

f

$\mathrm{t}$

$\mathrm{T}$

$\mathrm{Ha} \quad$ local magnetic field parameter

c free stream flow rate

$\mathrm{T}_{\mathrm{f}} \quad$ hot fluid temperature

$\mathrm{h}_{\mathrm{f}} \quad$ heat transfer coefficient

A unsteadiness parameter

$\mathrm{k}$ thermal conductivity

coefficient

$\mathrm{U}_{\infty} \quad$ free stream temperature

$\mathrm{m} / \mathrm{s}$

\section{Greek Symbols}

$\Psi \quad$ stream function

$\theta \quad$ dimensionless temperature

$\mu \quad$ dynamic viscosity

$\alpha \quad$ thermal diffusivity

$\eta \quad$ similarity variable

$\lambda$ unsteadiness parameter

$\rho \quad$ fluid density

u kinematic viscosity

$\sigma \quad$ fluid electrical conductivity

$\delta \quad$ Navier slip parameter

\section{REFERENCES}

Ali, M. E. and Magyari, E., Unsteady fluid and heat flow induced by a submerged stretching surface while its steady motion is slowed down gradually. Int. J. Heat \& Mass Trans., Vol. 50, 188-195 (2007).

Bhattacharyya, K., Mukhopadhyay, S. and Layek, G. C., MHD boundary layer slip flow and heat transfer over a flat plate. Chin. Phys. Lett., Vol. 28, No. 2, 024701 (2011).

Beg, A. O., Bakier, A. Y., Prasad, V. R., Zueco, J., and Ghosh, S. K., Nonsimilar, laminar, steady, electrically-conducting forced convection liquid metal boundary layer flow with induced magnetic field effects. Int. J. Thermal Sc., Vol. 48, (8): 1596-1606 (2009).

Chamkha, A. J. and Khaled, A. A., Similarity solutions for hydromagnetic mixed convection heat and mass transfer for Hiemenz flow through porous media. Int. J. Numer. Meth. Heat Fluid Flow, 10, (1), 94-115 (2000).

Chaudhary, R. C. and Kumar Jha, A., Heat and mass transfer in elastico-viscous fluid past in impulsively started infinite vertical plate with hall effect. Latin American Applied Research, 38, 1726 (2008).

Choi, C. H., Westin, K. J. A. and Breuer, K. S., To slip or not to slip water flows in hydrophilic and hydrophobic micro-channels. Proceedings of IMECE, New Orleans, Louisiana (2002).

Dulal, P. and Hiremath, P. S., Computational modeling of heat transfer over an unsteady stretching surface embedded in a porous medium. Meccanica, 45, (3), 415-524 (2009).

Huang, P. and Breuer, K. S., Direct measurement of slip length in electrolyte solutions, Physics of Fluids, Vol. 19, 028104 (2007).

Kumar, R. and Chand, K., Effect of slip conditions and hall current on unsteady MHD flow of a viscoelastic fluid past an infinite vertical porous plate through porous medium. International Journal of Engineering Science and Technology (IJEST), Vol. 3 No. 4, pp. 3124-3123 (2011).

Makinde, O. D., On MHD boundary-layer flow and mass transfer past a vertical plate in a porous medium with constant heat flux. Int. J. Num. Methods for Heat \& Fluid Flow, Vol. 19, Nos. 3/4, 546-554 (2009).

Makinde, O. D. and Ogulu, A., The effect of thermal radiation on the heat and mass transfer flow of a variable viscosity fluid past a vertical porous plate permeated by a transverse magnetic field. Chemical Engineering Communications, Vol. 195, No. 12, 1575 -1584 (2008).

Makinde, O. D., Similarity solution of hydromagnetic heat and mass transfer over a vertical plate with a convective surface boundary condition. International Journal of Physical Sciences, Vol. 5, (6), 700-710 (2010).

Matthews, M. T. and Hill, J. M., A note on the boundary layer equations with linear slip boundary condition. Applied Mathematics Letters, 21, 810-813 (2008).

Nachtsheim, P. R. and Swigert, P., Satisfaction of the asymptotic boundary conditions in numerical solution of the system of nonlinear equations of boundary layer type. NASA TND-3004 (1965).

Navier, C. L. M. H., Mémoire sur les lois du mouvement des fluides. Mém. Acad. R. Sci. Inst. France 6, 389-440 (1823).

Pit, R., Hervet, H. and Leger, L., Direct experimental evidence of slip in hexadecane: solid interfaces. Phys. Rev. Lett., 85, 980-983 (2000). 
Singh, A. K., and Singh, N. P., Heat and mass transfer in MHD flow of a viscous fluid past a vertical plate under oscillatory suction velocity. Indian J. Pure and Appl. Math., 34, 429- 442 (2003).
Vajravelu, K. and Nayfeh, J., Hydromagnetic flow of a dusty fluid over a stretching sheet. International Journal of Non-linear Mechanics, Vol. 27, (6), 937-945 (1992). 